\section{Nuanced eosinophils}

Infection with Aspergillus fumigatus can lead to aspergillosis in immunocompromised people as well as allergic asthma in atopic patients. In PLoS Pathogens, Levitz and colleagues investigate the role of eosinophils in models of $\boldsymbol{A}$. fumigatus infection and allergy. Pulmonary eosinophils interact with $A$. fumigatus conidia, which results in the killing of these fungi. The activity of eosinophils is critical, as their absence results in much greater mortality after infection with a virulent strain of $A$. fumigatus. Unexpectedly, after infection or induction of allergic asthma, eosinophils are also a chief source of the inflammatory cytokines IL-17 and IL-23, and in fact, under the conditions assessed, they are almost exclusively the producers of IL-23 in the lungs. The main effect of this cytokine secretion is probably to drive the recruitment and/or local proliferation of inflammatory monocytes and lung macrophages. Therefore, in addition to being simply effector cells, lung eosinophils are probably important in shaping the local immune response. ZF PLoS Pathog. (17 January 2017) http://dx.doi.org/10.1371/ journal.ppat. 1006175

\section{Inflammasomes in human aging}

'Inflammaging' describes the chronic low-grade inflammatory process that occurs with aging and its associated diseases. In Nature Medicine, Furman et al. investigate the Stanford-Ellison longitudinal cohort to determine the contribution of inflammasome signatures and activity to inflammaging. Specific inflammasome expression modules correlate significantly with age and age-related diseases such as hypertension, as well as with diminished longevity. Inflammasome expression is also associated with oxidative stress, metabolic dysfunction and the presence of higher concentrations of particular metabolites in the blood. Two of these metabolites, adenine and $\mathrm{N}^{4}$-acetylcytidine, prime and/or activate the NLRC4 inflammasome and induce hypertension and inflammatory signatures when administered together to mice. Inflammasomes may therefore be important targets in normalizing the inflammatory status and associated diseases of the elderly.

Nat. Med. (16 January 2017) http://dx.doi.org/10.1038/nm.4267

\section{Neuroimmune interactions: ILC3s}

The vagal system regulates the production of lipid mediators such as resolvins, which are linked to the resolution phase of inflammation. In Immunity, Serhan and colleagues show that after infection with Escherichia coli, the vagus nerve controls the resolution of inflammation by signaling through cholinergic receptors on peritoneal group 3 innate lymphoid cells (ILC3s). Resection of the right vagus trunk induces a decrease in the peritoneal concentration of the lipid mediator PCTR 1 and the number of peritoneal ILC3s. After infection with $E$. coli, mice whose vagus nerve has been cut show enhanced infiltration of neutrophils, diminished phagocytosis by macrophages and delayed resolution of inflammation. Administration of PCTR1 or transfer of ILC3s rescues mice from these effects. ILC3s express cholinergic receptors and in response to acetylcholine, a vagusnerve-derived neurotransmitter, induce the production of PCTR1 in peritoneal macrophages.

Immunity 46, 92-105 (2017)

\section{Neuroimmune interactions: astrocytes}

Reactive (A1) astrocytes are induced in neuroinflammation and upregulate their expression of genes encoding components of the classic complement cascade, known to be destructive to synapses. In Nature, Barres and colleagues show that A1 astrocytes are induced by activated microglia. The effect is mediated by the pro-inflammatory mediators IL-1 $\alpha$, TNF and C1q, which can induce A1 astrocytes directly. In contrast to wild-type mice, $C s f 1 r^{-1}$ mice (which lack microglia) and Ill $\mathrm{a}^{-/-} \mathrm{Tnf}^{--} \mathrm{Clqa}^{-/-}$mice do not generate A1 astrocytes after treatment with lipopolysaccharide. A1 astrocytes are impaired in their ability to induce synapse formation and to phagocytose myelin and kill various types of central-nervous-system neurons and mature oligodendrocytes in co-culture assays. Astrocytes positive for the complement component $\mathrm{C} 3$, which is considerably upregulated in A1 astrocytes, are detected in brain tissue from patients with neurodegenerative conditions such as Alzheimer's, Parkinson's or Huntington's disease or multiple sclerosis, in close association with $\mathrm{CD} 68^{+}$microglia or macrophages.

Nature 541, 481-487 (2017)

\section{Anti-dengue IgG1}

Patients re-infected with dengue virus of a serotype different from that of the initial infection are thought to be at risk of developing severe dengue hemorrhagic fever, due to the production of nonneutralizing antibodies to the second dengue virus. In Science, Ravetch and colleagues report alterations in the glycosylation pattern of anti-viral immunoglobulin G1 (IgG1) in those patients who develop severe disease after re-infection relative to the pattern in other patients who experience a milder disease. Loss of IgG1 fucosylation and increased ratios of IgG1 to IgG2 correlate with the platelet loss (thrombocytopenia) that characterizes severe dengue disease. 'Humanized' mice receiving patients' afucosylated IgG1, which binds with higher affinity to activating Fc $\gamma$ RIII receptors than does fucosylated IgG1, experienced Fc $\gamma R$-dependent platelet loss. Furthermore, IgG1 that recognizes dengue NS1 protein cross-reacts with platelet proteins. Thus, disease severity is linked to cross-reactive IgG1 with afucosylated Fc regions that recognize Fc $\gamma \mathrm{RIII}$ with great avidity. $L A D$ Science 355, 395-398 (2017)

\section{CyTOF analysis of anti-tumor responses}

Patients with cancer are increasingly treated by immunotherapy, yet some tumors remain refractory to such therapies. In Cell, Spitzer et al. utilize mass cytometry to perform systems analysis of effective antitumor responses in a mouse model of spontaneous triple-negative breast cancer, a tumor type that resists checkpoint blockade directed against the immunoinhibitory receptor PD-1. Effective responses are elicited by intratumoral injection of allogeneic immunoglobulin, antibody to the costimulatory receptor CD40 and interferon- $\gamma$. Enduring anti-tumor responses require the activation and proliferation of immune cells in both tumor sites and 'systemic' sites, including activation of B cells and $\mathrm{CD}^{+}{ }^{+} \mathrm{T}$ cells in lymphoid tissues. Surprisingly, a dominant component of this effective response is $\mathrm{CD} 90^{+} \mathrm{CD} 4^{+} \mathrm{T}$ cells rather than $\mathrm{CD}^{+} \mathrm{T}$ cells. Patients with melanoma who respond to antibody to the immunomodulatory receptor CTLA-4 likewise have a greater frequency of activated $\mathrm{CD}^{+} \mathrm{T}$ cells than that of non-responders. Such findings should inform the design of future clinical trials.

$L A D$ Cell 168, 487-502 (2017) 